\title{
Impact of an inpatient multidisciplinary glucose control management program
}

\author{
Denise P. Momesso', Rubens Carmo Costa Filho', João Luiz Ferreira Costa', \\ Felipe Saddy', Ayla Mesquita', Marcela Calomeni', Claudia dos Santos Silva', \\ Jacqueline Farret', Mariana Leon Vasques', Aline G. Santos', \\ Ana Paula Vieira Cabral', Dayane Ribeiro', Luciana Reis', \\ Maria de Fátima M. Muino', Roberta Santiago Vitorino', \\ Claudio Amorim Monteiro', Evandro Tinoco', Andre Volschan'
}

${ }^{1}$ Hospital Pró-Cardíaco, Rio de Janeiro, RJ, Brasil

\section{Correspondence to:} Denise Momesso Rua General Polidoro, 192 22280-003 - Rio de Janeiro, RJ, Brazil endocrinologia@denisemomesso.com.b

Received on Nov/8/2017 Accepted on June/13/2018

DOI: 10.20945/2359-39970000000071

\begin{abstract}
Objective: Glycemic control has been increasingly recognized as a critical element in inpatient care, but optimal management of blood glucose in the hospital setting remains challenging. The aims of this study were to describe and evaluate the impact of the implementation of an inpatient multidisciplinary glucose control management program on glucose control in hospitalized patients. Materials and methods: Retrospective analysis of medical records and glucose monitoring data obtained by pointof-care testing (POCT) in hospitalized patients before (May 2014) and after (June 2015 and May 2017) the implementation of the program. Results: We analyzed 6888, 7290, and 7669 POCTs from 389, 545, and 475 patients in May 2014, June 2015, and May 2017, respectively. Hyperglycemia ( $\geq 180 \mathrm{mg} /$ $\mathrm{dL}$ ) occurred in 23.5\%, 19.6\%, and 19.3\% POCTs in May 2014, June 2015, and May/2017, respectively ( $p<0.001$ ), while severe hyperglycemia ( $\geq 300 \mathrm{mg} / \mathrm{dL}$ ) was observed in $2.5 \%, 2.2 \%$, and $1.8 \%$ of them, respectively $(p=0.003)$. Hyperglycemia $(\geq 180 \mathrm{mg} / \mathrm{dL}$ ) reduced significantly from May 2014 to June 2015 (16.3\%, p < 0.001) and from May 2014 to May 2017 (17.8\%, p < 0.001). No significant changes occurred in hypoglycemic parameters. Conclusions: The implementation of an inpatient multidisciplinary glucose control management program led to significant reductions in hyperglycemic events. The key elements for this achievement were the development of institutional inpatient glycemic control protocols, establishment of a multidisciplinary team, and continuing educational programs for hospital personnel. Altogether, these actions resulted in improvements in care processes, patient safety, and clinical outcomes of hospitalized patients. Arch Endocrinol Metab. 2018;62(5):514-22
\end{abstract}

Keywords

Hyperglycemia; hypoglycemia; inpatient glucose control; diabetes mellitus; systems, point-of-care

\section{INTRODUCTION}

G lycemic control has been increasingly recognized I as a critical element in inpatient care (1-7). Several lines of evidence corroborate the concept that both hyperglycemia and hypoglycemia are associated with adverse outcomes to the patient. Hyperglycemia may occur in hospitalized patients with known diabetes or acutely ill individuals with previously normal glucose tolerance ("stress hyperglycemia") due to increased circulating counterregulatory hormones in response to stress. Irrespective of the cause, hyperglycemia on an inpatient setting is an independent marker of increased morbidity and mortality. During treatment of hyperglycemia, a major concern is the occurrence of hypoglycemia, which is also an independent risk factor for poor clinical outcomes (8-15). Therefore, great emphasis has been placed on optimizing the treatment of hospitalized patients with diabetes and hyperglycemia.

Based on data from multiples studies and clinical trials, the management of hyperglycemia in a hospital setting has recently evolved (16-23). Current consensus statements from the American Diabetes Association, American Association of Clinical Endocrinologists, Endocrine Society, and Brazilian Diabetes Association have recommended therapy of critically ill patients with persistent hyperglycemia, starting at a blood glucose level of $180 \mathrm{mg} / \mathrm{dL}$; once insulin is started, the therapeutic glucose target should be within the 140$180 \mathrm{mg} / \mathrm{dL}$ range (1-6). In noncritical care patients, the recommended values are $<140 \mathrm{mg} / \mathrm{dL}$ for fasting glucose and $<180 \mathrm{mg} / \mathrm{dL}$ for random glucose, and 
the preferable regimen is the use of a basal insulin along with premeal and supplemental insulin, instead of sliding scale insulin. Optimal glycemic control also includes the prevention of hypoglycemia (1-6,24-26). Reducing the variability in glucose levels may also be important in improving outcomes $(27,28)$.

Despite existing evidence, the optimal glucose management in a hospital setting remains challenging, as the achievement of improved glycemic control in a hospital setting meets numerous obstacles. In this scenario, the American College of Endocrinology, the American Diabetes Association, and the Brazilian Diabetes Society have released calls to action outlining strategies for a successful implementation of inpatient glucose control management programs $(2,5,7)$. Systembased key issues outlined included the need for the development and evaluation of: 1) clinical protocols to guide management, clinical decisions, and prescriptions; 2) multidisciplinary glucose control management teams; and 3 ) provider-delivered educational programs to improve knowledge and address barriers to achieving glycemic control $(2,5,7,29,30)$.

The aims of this study were to describe and evaluate the impact of the implementation of a multidisciplinary glucose control management program for hospitalized patients. The study was conducted to determine if such program would improve inpatient safety by reducing the number of hyperglycemic events.

\section{MATERIALS AND METHODS}

The study was conducted at the Pró-Cardiaco Hospital, a 99-bed, tertiary-care, medical and surgical center located in Rio de Janeiro, Brazil. The medical center comprises an emergency room, two adult intensive care units (ICU), one coronary care unit, one surgical intensive care unit, one surgical semi-intensive care unit, three clinical semi-intensive units, one oncology care unit, and one day clinic. The hospital has an extensive referral network and includes outpatient specialty treatment and imaging centers, clinics, and rehabilitation centers.

Since 2001, the ICUs of the hospital have developed a protocol of insulin treatment for critically ill patients. In 2012, we introduced a hospital-wide inpatient multidisciplinary glucose control management program (MGCP) to facilitate the development of uniform glucose management policies and staff education based on current clinical practice guidelines. The main hallmarks of this program were the development of an Institutional inpatient glycemic control protocols in January 2012, and the establishment of a multidisciplinary glycemic control team in June 2014.

\section{Institutional inpatient glycemic control protocols}

In 2012, institutional glycemic control protocols were developed in an effort to improve and standardize the glycemic control of hospitalized patients. These protocols are aligned with international and local recommendations (2-6) and are frequently revised and updated based on these recommendations and local assistance requirements.

According to the institutional protocol, blood glucose levels of all critical and noncritical care patients admitted to the hospital are monitored using pointof-care testing (POCT). Glucose monitoring may be suspended 72 hours after admission of noncritically ill patients and in those without diabetes or current illness, or not using medications associated with hyperglycemia or hypoglycemia. Monitoring may also be suspended in patients whose blood glucose measurements have been within the normal range for 72 hours. All results obtained by POCT are downloaded directly from the glucometer (Precision $^{\circledR}$, Abbott Diabetes Care Inc., Alameda, CA, USA) to the patient's electronic medical records. In case of glucose measurements $\leq 40$ $\mathrm{mg} / \mathrm{dL}$ or $\geq 300 \mathrm{mg} / \mathrm{dL}$, a direct notification is sent via e-mail from the medical record to the endocrinologist in charge of the patient.

Insulin therapy is the method of choice for glycemic control in hospitalized patients with hyperglycemia. The institutional protocol recommends the discontinuation of antidiabetic drugs for most patients upon hospital admission for acute illness. Patients with type 1 or 2 diabetes receiving insulin as multiple daily injections require treatment with basal-bolus insulin regimens, and their insulin doses are modified according to the patient's clinical status.

In noncritical care patients, the glycemic targets set by the institutional protocol are $<140 \mathrm{mg} / \mathrm{dL}$ before meals and $<180 \mathrm{mg} / \mathrm{dL}$ for random glucose measurements. In patients with terminal illness and/ or limited life expectancy, the glycemic target is < $180 \mathrm{mg} / \mathrm{dL}$. In noncritical care patients, POCTs are performed based on the timing of the meals: before meals in patients receiving an oral diet, every 6 hours in those with continuous enteral or parenteral nutrition, and every 4 hours in patients not receiving diet. Patients 
with hyperglycemia (glucose level $\geq 200 \mathrm{mg} / \mathrm{dL}$ ) undergo more frequent glucose measurements for detection and treatment of hyperglycemia, prevention of hypoglycemia following supplemental insulin administration, and prevention of glycemic variability. The protocol recommends a basal-bolus insulin regimen, including a basal component with a longacting insulin analogue (glargine or detemir) or intermediate-acting insulin (NPH) once or twice daily, and a bolus component with ultra-rapid-acting insulin (lispro) administered according to meals and supplemental doses according to glycemic levels. Ultrarapid insulin analogues are the insulin of choice for the bolus component of the regimen at our institution, based on evidence in the literature showing better glycemic control in hospitalized patients with this type of insulin when compared with regular insulin, with a lower number of hypoglycemic episodes (24). The total dose of insulin administered is individualized and based on the patient's previous insulin regimen, glycemic levels, total body weight, clinical status, and nutritional therapy. Basal insulin is administered to all patients with previous insulin regimens and in those with sustained hyperglycemia. In insulin-naïve patients, the recommended initial total daily insulin dose is 0.2 $0.5 \mathrm{U} / \mathrm{kg} /$ day, with approximately $50 \%$ of the dose administered as basal insulin (preferably glargine) and the remainder as bolus insulin. Bolus insulin contemplates the patient's diet and carbohydrate intake, as follows: bolus insulin before meals in patients on an oral diet, every 6 hours in those on continuous enteral or parenteral nutrition therapy, and 3 to 4 times a day before meals in patients receiving cyclic enteral nutrition. The supplemental component of bolus insulin is administered according to the patient's glucose level. Our institution has five different supplemental insulin regimens: (i) usual insulin dose; (ii) reduced insulin dose, recommended for patients at risk for hypoglycemia; (iii) increased insulin dose, recommended for patients with insulin resistance; (iv) a regimen for patients with terminal illness and/or limited life expectancy; and (v) a regimen for patients with no oral or enteral nutritional therapy (fasting).

For critical care patients, blood glucose levels are measured at one-hour intervals. Insulin therapy is started in critically ill patients with sustained hyperglycemia, defined as at least two glucose measurements $\geq 180$ $\mathrm{mg} / \mathrm{dL}$, using continuous intravenous regular insulin infusion. In patients with continuous intravenous insulin infusion, the glycemic targets are $140-180 \mathrm{mg} / \mathrm{dL}$, and glucose levels $<100 \mathrm{mg} / \mathrm{dL}$ should be avoided. Insulin infusion is adjusted every hour according to glucose levels. The continuous intravenous insulin infusion protocol used at the institution was adapted from the Yale Insulin Infusion Protocol for critically ill patients, as previously described (23).

\section{Multidisciplinary glycemic control team}

A multidisciplinary glycemic control team was created at our institution in June 2014. The main goal of its implementation was to develop a centralized multidisciplinary team to address barriers to achieving glycemic control in the hospital setting. The team is chaired by an endocrinologist and includes physicians (endocrinologist, intensivists, hospitalists, and house staff), nurse practitioners, pharmacists, dietitians, and POCT/laboratory medicine specialists. The team aims at promoting the correct implementation of protocols for management of hyperglycemia and hypoglycemia, educating physicians and nurses on the proper use of these protocols, performing continuous education of health care professionals, promoting clinical decision aids, and surveilling performance measures for quality improvement. The members of the team deliberate on regular monthly meetings and on a daily basis during continuous patient care.

\section{Education programs for hospital personnel}

The educational programs for hospital personnel are delivered on a regular basis and include the participation of nurses, house staff, physicians, pharmacists, and dietitians. Educational sessions are often offered in the hospital at different time periods to ensure delivery to as many staff members as possible. All staff members (physicians, nurses, pharmacists, and dietitians) are exposed to the educational program upon joining the hospital staff. Regular educational sessions are delivered at different hospital units (a total of 10 units) at a maximum interval of 6 months. Once weekly, the endocrinologist in charge delivers educational orientation to staff members during patient care (onsite training).

The main aspects outlined in these educational programs include the impact of inpatient glycemic control on patient care, introduction and reinforcement of protocols for management of hyperglycemia and hypoglycemia, information about patients at risk for 
hypoglycemia and hyperglycemia, identification of signs of hypoglycemia, characteristics of the different types of insulin and administration routes (intravenous or subcutaneous for basal insulin administration, prandial or correction doses), a review of insulin requirements during health and illness, inpatient use of antihyperglycemic agents, the influence of diet, the importance of respecting the appropriate time of glucose measurement, and proper documentation of patient treatment.

\section{Glucose monitoring by point-of-care testing}

Glucose monitoring by POCT was performed with the glucometers Precision Xceed Pro (PXP) ${ }^{\circledR}$ and FreeStyle Precision Pro (FSPP) ${ }^{\circledR}$ (Abbott Diabetes Care Inc., Alameda, CA, USA). In noncritical patients, glycemic measurements with POCT used capillary blood samples obtained by fingertip puncture after local hygiene, while in critical patients, venous or arterial blood samples were used instead. The same type of blood source was used in each patient according to his or her clinical status. Areas with edema, lesions, hypoperfusion, and/ or venous infusion routes with continuous infusion of solutions were avoided during blood drawing.

The glucometers underwent continuous quality control. All devices were calibrated every 24 hours with high and low glucose control samples; when calibration was not performed in 24 hours, the glucometer was automatically blocked from use or for POCT. All glucometers underwent a harmonization process every 6 months, consisting on a comparison of the results obtained by POCT with those obtained by the laboratory (Dimension $^{\circledR}$, Siemens Healthcare Diagnostics, Deerfield, IL, USA). During the study, the variation coefficient between the results obtained by the POCT and those by the laboratory was $<10.78 \%$ in all glucometers, which is aligned with standards of care (31).

\section{Glycemic control quality indicators}

Since the MGCP implementation, quality indicators of glycemic control, hypoglycemia, and hyperglycemia were monthly assessed using data from the POCTs. All results obtained by POCT were electronically downloaded directly from Abbott's Precision ${ }^{\circledR}$ glucometer to the software using the Abbott Precision Web System (Abbott Diabetes Care Inc., Alameda, CA, USA) and to the patient's electronic medical records, providing accurate data for the indicators. Calculation of the rates of hyperglycemia and hypoglycemia were as follows: (i) hyperglycemia $(\geq 180 \mathrm{mg} / \mathrm{dL}$ ) as the number of glucose measurements by POCT $\geq$ $180 \mathrm{mg} / \mathrm{dL}$ (numerator) divided by the total number of glucose measurements by POCT performed in that given period (denominator), (ii) severe hyperglycemia $(\geq 300 \mathrm{mg} / \mathrm{dL})$ as the number of glucose measurements by POCT $\geq 300 \mathrm{mg} / \mathrm{dL}$ (numerator) divided by the total number of glucose measurements by POCT performed in the period (denominator), (iii) hypoglycemia $(\leq 70 \mathrm{mg} / \mathrm{dL})$ as the number of glycemic measurement by POCT $\leq 70 \mathrm{mg} / \mathrm{dL}$ (numerator) divided by the total number of glycemic measurement by POCT performed in the period (denominator), (iv) severe hypoglycemia $(\leq 40 \mathrm{mg} / \mathrm{dL})$ as the number of glycemic measurement by POCT $\leq 40 \mathrm{mg} / \mathrm{dL}$ (numerator) divided by the total number of glycemic measurement by POCT performed in the period (denominator).

Adherence to the institutional inpatient glycemic protocols was measured regularly by a revision of the patient's prescriptions and medical records.

\section{Data collection}

We performed a retrospective analysis of the medical records of the patients admitted to the hospital. We analyzed the data obtained in May 2014, before the MGCP implementation, and in June 2015 and May 2017, after the MGCP implementation.

The inclusion criteria were all critical and noncritical patients admitted to the hospital, aged $\geq 18$ years, who had blood glucose measured by POCT by Abbott's Precision ${ }^{\circledR}$ glucometer according to the institutional protocol, and a length of stay $\geq 2$ days. The exclusion criteria were age $<18$ years, length of stay shorter than 2 days, and admissions limited to the emergency room or to the day-clinic unit. We also excluded the results of POCT glucose monitoring obtained during surgical procedures.

We analyzed the quality indicators of glycemic control and the results of glucose monitoring obtained by POCT before the implementation of the MGCP in May 2014 and after the implementation of the MGCP in June 2015 and May 2017. Based on blood glucose levels, the patients were characterized as having hyperglycemia $(\geq 180 \mathrm{mg} / \mathrm{dL})$, severe hyperglycemia $(\geq 300 \mathrm{mg} / \mathrm{dL})$, hypoglycemia $(\leq 70 \mathrm{mg} / \mathrm{dL})$, or severe hypoglycemia $(\leq 40 \mathrm{mg} / \mathrm{dL})$. The rates of hyperglycemia $(\geq 180 \mathrm{mg} / \mathrm{dL}$ ), severe hyperglycemia 
( $\geq 300 \mathrm{mg} / \mathrm{dL})$, hypoglycemia $(\leq 70 \mathrm{mg} / \mathrm{dL})$, and severe hypoglycemia $(\leq 40 \mathrm{mg} / \mathrm{dL})$ were calculated as described above.

Adherence to the institutional inpatient glycemic protocols was also analyzed by a revision of the patients' prescriptions and medical records in May 2014, June 2015, and May 2017. A prescription was considered to be not compliant to the institutional protocols if inadequate to the diet or clinical scenario. Inadequacy with the diet was present when the prescription was not conformed with the type of diet (i.e., oral, enteral, and parenteral diets, or fasting), not coordinated with the POCT, or when insulin was not administered around mealtime. Inadequacy with the clinical scenario occurred when the insulin prescribed was not suitable for the patient's clinical status according to the institutional protocol (i.e., intravenous insulin for critical patients, subcutaneous insulin for noncritical patients, basal-bolus insulin regimen for noncritical patients with sustained hyperglycemia and/or previous use of a basal-bolus insulin regimen, or supplemental subcutaneous insulin for noncritical patients in accordance to patients characteristics (usual insulin dose, risk of hypoglycemia, insulin resistance, terminal illness, and/or limited life expectancy).

The local institutional ethics committee approved the study.

\section{Statistical analysis}

Continuous data are presented as mean and standard deviation values or median values and range. Comparisons of categorical variables were performed with the Fisher's exact or chi-square test, while continuous variables were compared using Student's t test.

The statistical analyses were performed with the software programs SPSS, version 20 (SPSS Inc., Chicago, IL, USA), Minitab 16, and Excel Office 2010. Statistical significance was set at $\mathrm{p}<0.05$.

\section{RESULTS}

The clinical characteristics of the hospitalized patients undergoing glucose monitoring in May 2014, June 2015, and May 2017 are described in Table 1. The groups had comparable baseline clinical characteristics except for age, which differed among the groups.

We analyzed 6888, 7290, and 7669 POCTs from 389,545 , and 475 patients in May 2014, June 2015, and May 2017, respectively. The mean number of glucose measurements per patient was $2.39 \pm 1.96$, $2.39 \pm 2.26$, and $3.64 \pm 2.76$ in May 2014, June 2015, and May 2017, respectively. There was a significant increase in glucose monitoring from May 2014 to May 2017 ( $\mathrm{p}=0.007$ ), but no differences between May 2014 and June 2015 ( $\mathrm{p}=0.99$ ).

Table 2 describes the quality indicators of inpatient glycemic control. In May 2014, June 2015, and May 2017, the rates were $23.5 \%, 19.6 \%$, and $19.3 \%$, respectively, for hyperglycemia $(\geq 180 \mathrm{mg} / \mathrm{dL} ; \mathrm{p}<$ 0.001 ), and $2.5 \%, 2.2 \%$, and $1.8 \%$, respectively, for severe hyperglycemia $(\geq 300 \mathrm{mg} / \mathrm{dL} ; \mathrm{p}=0.003)$.

Table 1. Baseline clinical characteristics of hospitalized patients with glucose measured by point-of-care testing (POCT)

\begin{tabular}{|c|c|c|c|c|}
\hline & May 2014 & June 2015 & May 2017 & p value \\
\hline $\begin{array}{l}\text { Number of patients undergoing } \\
\text { POCT }\end{array}$ & 389 & 545 & 475 & \\
\hline Age (years) & $72.7 \pm 16.6$ & $73.0 \pm 16.2$ & $75.6 \pm 14.6$ & $0.04^{*}$ \\
\hline \multicolumn{5}{|l|}{ Admission } \\
\hline Clinical patients & $80.8 \%$ & $83.9 \%$ & $78.5 \%$ & 0.086 \\
\hline Surgical patients & $19.2 \%$ & $16.1 \%$ & $21.5 \%$ & \\
\hline \multicolumn{5}{|l|}{$\begin{array}{l}\text { Patients' characteristics at } \\
\text { admission }\end{array}$} \\
\hline Critical care & $10.4 \%$ & $13.4 \%$ & $9.7 \%$ & 0.141 \\
\hline Noncritical care & $89.6 \%$ & $86.6 \%$ & $90.3 \%$ & \\
\hline Diabetes mellitus & $33.7 \%$ & $30.4 \%$ & $35.1 \%$ & 0.176 \\
\hline $\begin{array}{l}\text { Rate of hyperglycemia } \\
\text { ( } \geq 180 \mathrm{mg} / \mathrm{dL} \text { ) on admission }\end{array}$ & $22.1 \%$ & $18.9 \%$ & $19.6 \%$ & 0.465 \\
\hline Length of stay (days) & $5(2-919)$ & $5(2-919)$ & $5(2-967)$ & 0.98 \\
\hline Intravenous insulin protocol & $7.3 \%$ & $8.8 \%$ & $6.5 \%$ & 0.371 \\
\hline
\end{tabular}

Data are expressed as mean \pm standard deviation, mean (range), or percentage. ${ }^{*}$ Statistically significant $(p<0.05)$. 
The rates of hyperglycemia $(\geq 180 \mathrm{mg} / \mathrm{dL})$ reduced significantly from May 2014 to June 2015 (16.3\%, $\mathrm{p}<0.001$ ) and from May 2014 to May 2017 (17.8\%, $\mathrm{p}<0.001)$. Similarly, the rates of severe hyperglycemia $(\geq 300 \mathrm{mg} / \mathrm{dL})$ reduced statistically significantly from May 2014 to May 2017 (28.5\%, p = 0.003), but were non-statistically significant between May 2014 and June $2015(13.6 \%, \mathrm{p}=0.175)$ (Figures 1 and 2).

There was no statistically significant change in hypoglycemic parameters over time (Table 2). Rates of

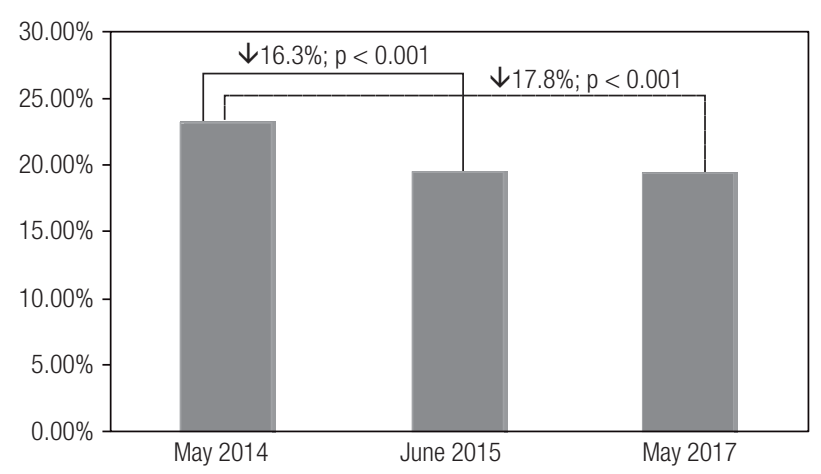

Figure 1. Rates of hyperglycemia ( $\geq 180 \mathrm{mg} / \mathrm{dL}$ ) in hospitalized patients in May 2014, June 2015, and May 2017.

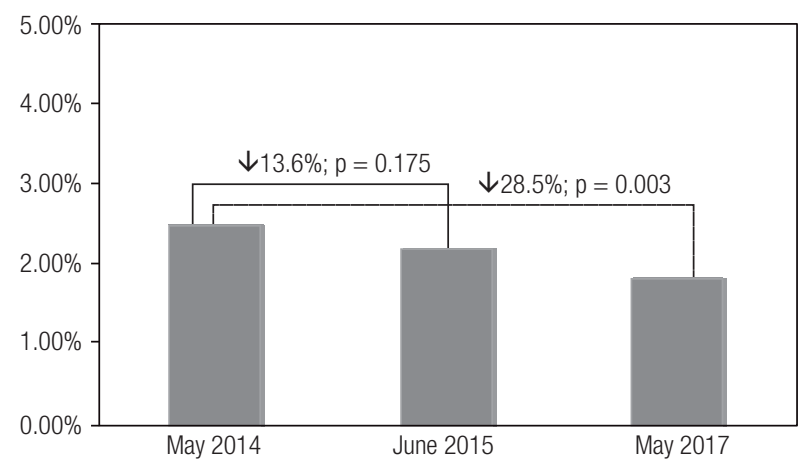

Figure 2. Rates of severe hyperglycemia ( $\geq 300 \mathrm{mg} / \mathrm{dL}$ ) among hospitalized patients in May 2014, June 2015, and May 2017. hypoglycemia $(\leq 70 \mathrm{mg} / \mathrm{dL}$ ) were $0.9 \%, 1.8 \%$, and $1.0 \%$ in May 2014, June 2015, and May 2017, respectively $(\mathrm{p}=0.710)$, while the rates of severe hypoglycemia $(\leq 40 \mathrm{mg} / \mathrm{dL})$ showed a non-statistically significant decrease of 34.3\% from May 2014 to May 2017 $(\mathrm{p}=0.336)$.

Table 3 describes the rates of adherence to the institutional inpatient glycemic protocols. Adherence to the protocol improved over time. The proportion of prescriptions not compliant with the institutional protocols decreased from $34.2 \%$ in May 2014 to $10.1 \%$ in June 2015, and 7.5\% in May 2017 ( $\mathrm{p}<0.001$ ). We found significant decreases in diet and clinical scenario inadequacies over time from May 2014 to May 2017.

\section{DISCUSSION}

The implementation of an inpatient multidisciplinary glucose control management program (MGCP) had a positive impact on glycemic control in hospitalized patients at our center. We observed that the key elements for this achievement were the implementation

Table 3. Rates of adherence to the institutional inpatient glycemic protocols

\begin{tabular}{lcccc}
\hline & $\begin{array}{c}\text { May } \\
\mathbf{2 0 1 4}\end{array}$ & $\begin{array}{c}\text { June } \\
\mathbf{2 0 1 5}\end{array}$ & $\begin{array}{c}\text { May } \\
\mathbf{2 0 1 7}\end{array}$ & p value \\
\hline $\begin{array}{l}\text { Prescriptions } \\
\text { not compliant } \\
\text { with the } \\
\text { institutional } \\
\text { protocols }\end{array}$ & $34.2 \%$ & $10.1 \%$ & $7.5 \%$ & $<0.001^{*}$ \\
$\begin{array}{l}\text { Noncompliance } \\
\text { with clinical }\end{array}$ & $20.0 \%$ & $6.3 \%$ & $5.2 \%$ & $0.026^{*}$ \\
$\begin{array}{l}\text { scenario } \\
\text { Noncompliance }\end{array}$ & $17.4 \%$ & $3.8 \%$ & $2.5 \%$ & $0.006^{\star}$ \\
with diet & & & & \\
\hline
\end{tabular}

Data are expressed as percentage. * Statistically significant $(p<0.05)$.

Table 2. Quality indicators of inpatient glycemic control

\begin{tabular}{|c|c|c|c|c|}
\hline & May 2014 & June 2015 & May 2017 & $\mathrm{p}$ value \\
\hline Number of patients undergoing POCT & 389 & 545 & 475 & - \\
\hline Number of glucose readings with POCT & 6888 & 7290 & 7669 & - \\
\hline Glucose level - mean (SD) (mg/dL) & $158.9 \pm 60.7$ & $150.5 \pm 59.1$ & $150.3 \pm 57.8$ & $<0.001^{*}$ \\
\hline Glucose level - median (IQR) (mg/dL) & $147.0(118-188)$ & $138.0(1120-177)$ & $137.0(109-178)$ & $<0.001^{*}$ \\
\hline Rate of severe hypoglycemia ( $\leq 40 \mathrm{mg} / \mathrm{dL}$ ) & $0.2 \%(n=12)$ & $0.1 \%(n=7)$ & $0.1 \%(n=9)$ & 0.336 \\
\hline Rate of hypoglycemia ( $\leq 70 \mathrm{mg} / \mathrm{dL}$ ) & $0.9 \%(n=65)$ & $1.8 \%(n=129)$ & $1.0 \%(n=79)$ & 0.710 \\
\hline Rate of hyperglycemia ( $\geq 180 \mathrm{mg} / \mathrm{dL}$ ) & $23.5 \%(n=1620)$ & $19.6 \%(n=1428)$ & $19.3 \%(n=1521)$ & $<0.001^{*}$ \\
\hline Rate of severe hyperglycemia ( $\geq 300 \mathrm{mg} / \mathrm{dL}$ ) & $2.5 \%(n=175)$ & $2.2 \%(n=160)$ & $1.8 \%(n=143)$ & $0.003^{*}$ \\
\hline
\end{tabular}

SD: standard deviation; IQR: interquartile range; POCT: point-of-care testing. ${ }^{*}$ Statistically significant $(p<0.05)$. 
of institutional inpatient glycemic control protocols, establishment of a multidisciplinary glycemic control team, and continuous educational programs for hospital personnel. Altogether, these actions resulted in a significant reduction in hyperglycemic events and improved safety among inpatients.

The development of institutional inpatient glycemic control protocols was important in guiding initial management, clinical decisions, and prescriptions at our center. With the protocols, we were able to standardize our policies and the patients' glycemic control. Since the implementation of the institutional protocols, different educational programs were delivered for hospital staff training. These protocols need to be constantly reevaluated and updated based on newly available evidence in the literature and on local demands of patient care. Other centers have described similar improvements in clinical outcomes with the adoption of insulin protocols for glucose management in critical and noncritical patients $(17,18,23,29,30,31-33)$.

Considered alone, the implementation of the institutional inpatient glycemic control was probably not enough to improve the process of care. The protocol was implemented in January 2012, and in May 2014, we observed that a great proportion of the prescriptions were still not compliant to the protocol. We then hypothesized that the staff education programs should be optimized and further actions should be taken, including the implementation of a multidisciplinary glycemic control team and the dissemination of quality indicators of glucose control. Indeed, we found that the implementation of hospital-wide glucose policies was best facilitated by targeted educational programs and clinical decision support infrastructure to facilitate acceptance by the hospital personnel. We then observed a significantly increased adherence to the institutional inpatient glycemic control protocols over time, accompanied by improved quality indicators of glycemic control in June 2015 and May 2017.

The establishment of a centralized multidisciplinary glycemic control team was a core and critical element in the development of our inpatient glucose management program. Through regularly scheduled monthly meetings and a culture of collaboration and teamwork, the members of the team promoted the implementation of protocols, education interventions, clinical decision aids, performance measures, and quality indicators of glycemic control across continuous inpatient care. In fact, we observed significant reductions of $17.8 \%$ in the rate of hyperglycemia ( $\geq 180 \mathrm{mg} / \mathrm{dL}$ ) and $28.5 \%$ in the rate of severe hyperglycemia ( $\geq 300 \mathrm{mg} / \mathrm{dL}$ ) from May 2014 to May 2017, before and after the implementation of the multidisciplinary glycemic control team, respectively. These reductions in hyperglycemic events were already observed one year before (in June 2015), and improved even further in May 2017, suggesting a continuous improvement in patient care and quality outcomes.

Our educational programs focused on the major challenges to optimal glucose management. Similar to other centers, the main obstacles we encountered included unanticipated nutritional changes, poor coordination of the POCT with the administration of insulin around mealtime, unanticipated changes in clinical status or medications, use of medications associated with increased insulin resistance (such as glucocorticoids, often in variable and changing doses), failure by clinicians of making adjustments in glycemic therapy based on daily blood glucose patterns, prolonged use of sliding scale insulin as monotherapy, multiple system/organizational barriers such as lack of communication and/or deficient knowledge of diabetes management among providers and caregivers $(7,29,30,32,33)$. Notably, we demonstrated that a collaborative work of the nurses, dietitians, and physicians reduced the inadequacy of the prescription with the type of diet and improved the coordination of POCT and administration of insulin around mealtime.

The impact of the MGCP on hypoglycemic events in inpatients was less established. We observed a nonsignificant $34.3 \%$ reduction in the rate of severe hypoglycemia $(\leq 40 \mathrm{mg} / \mathrm{dL})$, which might be due to the low rate of such event at our center. The rates of hypoglycemia $(\leq 70 \mathrm{mg} / \mathrm{dL})$ were similar over time. Hypoglycemia is a possible unwanted consequence of improved control of hyperglycemia and may be associated with increased morbidity (3-5,21). Therefore, our results of reduced hyperglycemia without increased hypoglycemic events demonstrate that our institutional protocols were safe. Indeed, it has been demonstrated that the implementation of standardized insulin order sets with less strict glycemic targets and frequent glucose monitorization are associated with better glycemic control and produce expected benefits in terms of patient safety across different hospitals $(18,23,25,29,30,32,33)$.

Our study has limitations inherent to its retrospective, nonrandomized design and the absence of a concurrent control group. This study was intended to evaluate 
intermediary outcomes as a quality improvement for hospitalized patients, and we did not evaluate morbidity, mortality, or other important clinical outcome data other than the rates of hyperglycemia and hypoglycemia. Another limitation regarding the analysis of the glycemic data was the potential for an increased type I error (i.e., a false-positive result) due to clustering of POCT values within patients and increased monitoring frequency upon observation of a hyperglycemic event. Indeed, the number of glucose monitoring tests among patients with normal glucose values may affect the proportion of abnormal values. Nevertheless, according to our institutional protocol, patients with hyperglycemia have more frequent POCT than those with normal glucose values.

Finally, despite the decrease in hyperglycemia rates, they still require further reduction, and efforts will be made for this purpose. Despite the fact that our quality indicators of glycemic control seem to be aligned with those of other hospitals, hyperglycemia in hospitalized patients is still frequently observed (34). The creation of a national benchmarking process would be important for the development of best practices and improved management of inpatient hyperglycemia (35).

In conclusion, the implementation of an inpatient multidisciplinary glucose control management program at our center was associated with improved care process and clinical outcomes, demonstrated by continued reductions in rates of hyperglycemic events. The key elements for these achievements were the development of institutional inpatient glycemic control protocols, establishment of a multidisciplinary team, and continuing educational programs for hospital personnel. Therefore, our results suggest that an inpatient multidisciplinary glucose control management program increased the awareness of the value of treating hyperglycemia in hospitalized patients, representing an important feature for inpatient safety and quality improvement.

Acknowledgments: the authors are grateful to Antonio Sérgio Cordeiro da Rocha, Plinio Nascimento Gomes, Graziela Mendonça, Robertta Mendonça Macedo Pinheiro, Tatiana de Jesus Pereira, Monica Cabral, and Andrea Medeiros for their important assistance and skillful advice.

Disclosure: no potential conflict of interest relevant to this article was reported.

\section{REFERENCES}

1. Inzucchi SE. Management of hyperglycemia in the hospital setting. N Engl J Med. 2006;355:1903-11.
2. ACE/ADA Task Force on Inpatient Diabetes. American College of Endocrinology and American Diabetes Association consensus statement on inpatient diabetes and glycemic control. Endocr Pract. 2006;12:458-68.

3. Moghissi ES, Korytkowski MT, DiNardo M, Einhorn D, Hellman $R$, Hirsch IB, et al. American Association of Clinical Endocrinologists; American Diabetes Association. American Association of Clinical Endocrinologists and American Diabetes Association consensus statement on inpatient glycemic control. Diabetes Care. 2009;32:1119-31.

4. Umpierrez GE, Hellman R, Korytkowski MT, Kosiborod M, Maynard GA, Montori VM, et al. Management of hyperglycemia in hospitalized patients in non- critical care setting: an Endocrine Society Clinical Practice Guideline. J Clin Endocrinol Metab. 2012;97:16-38.

5. Controle da glicemia no paciente hospitalizado. Posicionamento oficial da Sociedade Brasileira de Diabetes $n^{\circ} 3 / 2015$. Available from: www.diabetes.org.br/profissionais.

6. Standards of Medical Care in Diabetes 2018. American Diabetes Association. Available from: http://care.diabetesjournals.org/content/41/Supplement_1.

7. Draznin B, Gilden J, Golden SH, Inzucchi SE; PRIDE investigators, Baldwin D, et al. Pathways to quality inpatient management of hyperglycemia and diabetes: a call to action. Diabetes Care. 2013;36:1807-14.

8. Krinsley JS. Association between hyperglycemia and increased hospital mortality in a heterogeneous population of critically ill patients. Mayo Clin Proc. 2003;78:1471-8.

9. Lipton JA, Barendse RJ, Van Domburg RT, Schinkel AF, Boersma $\mathrm{H}$, Simoons Ml, et al. Hyperglycemia at admission and during hospital stay are independent risk factors for mortality in high risk cardiac patients admitted to an intensive cardiac care unit. Eur Heart J Acute Cardiovasc Care. 2013;2:306-13.

10. Fuentes B, Castillo J, San José B, Leira R, Serena J, Vivancos J, et al.; Stroke Project of the Cerebrovascular Diseases Study Group, Spanish Society of Neurology. The prognostic value of capillary glucose levels in acute stroke: the Glycemia in Acute Stroke (GLIAS) study. Stroke. 2009;40:562-8.

11. Capes SE, Hunt D, Malmberg K, Gerstein HC. Stress hyperglycaemia and increased risk of death after myocardial infarction in patients with and without diabetes: a systematic overview. Lancet. 2000;355:773-8.

12. McAlister FA, Majumdar SR, Blitz S, Rowe BH, Romney J, Marrie TJ. The relation between hyperglycemia and outcomes in 2,471 patients admitted to the hospital with community-acquired pneumonia. Diabetes Care. 2005;28:810-5.

13. Egi M, Bellomo R, Stachowski E, French CJ, Hart G. Variability of blood glucose concentration and short-term mortality in critically ill patients. Anesthesiology. 2006;105:244-52.

14. Krinsley JS. Glycemic variability and mortality in critically ill patients: the impact of diabetes. J Diabetes SciTechnol. 2009;3:1292-301.

15. Malmberg K, Norhammar A, Wedel H, Ryden L. Glycometabolic state at admission: important risk marker of mortality in conventionally treated patients with diabetes mellitus and acute myocardial infarction: long-term results from the Diabetes and InsulinGlucose Infusion in Acute Myocardial Infarction (DIGAMI) study. Circulation. 1999;99:2626-32.

16. Furnary AP, Zerr KJ, Grunkemeier GL, Starr A. Continuous intravenous insulin infusion reduces the incidence of deep sternal wound infection in diabetic patients after cardiac surgical procedures. Ann Thorac Surg. 1999;67:352-60.

17. van den Berghe G, Wouters P, Weekers F, Verwaest C, Bruyninckx $F$, Schetz $M$, et al. Intensive insulin therapy in critically ill patients. N Engl J Med. 2001;345:1359-67.

18. Krinsley JS. Effect of an intensive glucose management protocol on the mortality of critically ill patients. Mayo Clinic Proc. 2004;79: 992-1000. 
19. Malmberg K, Rydén L, Wedel H, Birkeland K, Bootsma A, Dickstein $\mathrm{K}$, et al. Intense metabolic control by means of insulin in patients with diabetes mellitus and acute myocardial infarction (DIGAMI 2): effects on mortality and morbidity. Eur Heart J. 2005;26:650-61.

20. Van den Berghe G, Wilmer A, Hermans G, Meersseman W, Wouters PJ, Milants I, et al. Intensive insulin therapy in the medical ICU. N Engl J Med. 2006;354:449-61.

21. The NICE-SUGAR Study Investigators. Intensive versus Conventional Glucose Control in Critically III Patients. N Engl J Med. 2009;360:1283-97.

22. Griesdale DE, de Souza RJ, van Dam RM, Heyland DK, Cook DJ, Malhotra A, et al. Intensive insulin therapy and mortality among critically ill patients: a meta-analysis including NICE-SUGAR study. CMJA. 2009;180:821-7.

23. Shetty S, Inzucchi SE, Goldberg PA, Cooper D, Siegel MD, Honiden S. Adapting to the new consensus guidelines for managing hyperglycemia during critical illness: the updated Yale insulin infusion protocol. Endocr Pract. 2012;18:363-70.

24. Ko KJ, Tomor V, Nathanson BH, Bouchard JR, Aagren M, Dubois RW. Does type of bolus insulin matter in the hospital? Retrospective cohort analysis of outcomes between patients receiving analogue versus human insulin. Clin Ther. 2010;32:1954-66.

25. Umpierrez GE, Smiley D, Zisman A, Prieto LM, Palacio A, Ceron $M$, et al. Randomized study of basal-bolus insulin therapy in the inpatient management of patients with type 2 diabetes (RABBIT 2 trial). Diabetes Care. 2007;30:2181-6.

26. Hirsch IB. Sliding Scale Insulin, Time to stop sliding. JAMA. 2009;301:213-4.
27. Egi M, Bellomo R. Reducing glycemic variability in intensive care unite patients: a new therapeutic target? J Diabetes Sci Tecnol. 2009;3:1302-8.

28. Krinsley S. Glycemic variability and mortality in critically ill patients: the impact of diabetes. J Diabetes SciTechnol. 2009;3(6):1292-301.

29. Maynard GA, Holdych J, Kendall H, Harrison K, Montgomery PA, Kulasa K. Improving glycemic control safely in critical care patients: a collaborative systems approach in nine hospitals. Endocr Pract. 2017;23:583-93.

30. Maynard GA, Childers D, Holdych J, Kendall H, Hoag T, Harrison K. Improving Glycemic Control Safely in Non-Critical Care Patients: A Collaborative Systems Approach in Nine Hospitals. Jt Comm J Qual Patient Saf 2017;43:179-88.

31. Diretrizes para a gestão e garantia de qualidade dos testes laboratoriais remotos da Sociedade Brasileira de Patologia Clínica/Medicina Laboratorial (SBPC/ML) 2a edição; 2016; Editora Manole Ltda.

32. Munoz M, Pronovost P, Dintzis J, KemmererT, Wang NY, ChangYT, et al. Implementing and evaluating a multicomponent inpatient diabetes management program. Jt Comm J Qual Patient Saf. 2012;38:195-206.

33. Hermayer KL, Cawley P, Arnold P, Sutton A, Crudup J, Kozlowski L, et al. Impact of improvement efforts on glycemic control and hypoglycemia at a university medical center. J Hosp Med. 2009;4:331-9.

34. Cook CB, Kongable GL, Potter DJ, Abad VJ, Leija DE, Anderson $M$, et al. Inpatient glucose control: a glycemic survey of 126 US hospitals. J Hosp Med. 2009;4:E7-E14.

35. Goldberg PA, Bozzo JE, Thomas PG, Mesmer MM, Sakharova OV, Radford MJ, et al. "Glucometrics": assessing the quality of inpatient glucose management. Diabetes Technol Ther. 2006;8:560-9. 